\title{
Dynamics of Electron-Monopole System ${ }^{1}$
}

\author{
Yoichi Kazama \\ Randall Laboratory of Physics, The University of Michigan, \\ Ann Arbor, Michigan 48109
}

\section{INTRODUCTION}

In this paper I would like to discuss the dynamics of the electronmonopole system ${ }^{2}$ (Kazama et al., 1977; Kazama and Yang, 1977) in $U(1)$ gauge theory, namely, the scattering and the bound-state properties of this system, within the context of un-second-quantized relativistic quantum mechanics.

To begin the discussion, I must describe the two types of essential difficulties associated with this fascinating yet somewhat peculiar system, difficulties that have plagued physicists for a long time. We must resolve these difficulties in order to have a consistent theory of an electron-monopole system.

The first and the best-known difficulty is the so-called Dirac string (Dirac, 1931, 1948), i.e., the string of singularities of the potential $A_{\mu}(x)$ describing the field of a fixed monopole. Although the space around the monopole is spherically symmetric and without singularities, one can easily show that no singularity-free potential exists for this field. Take a sphere around a monopole and compute the total outward flux through it by the sum of the two line integrals of $A_{\mu}$ going around the equator in the opposite directions. If $A_{\mu}$ is nonsingular, Stokes' theorem guarantees that this is a valid way of computing the flux. But one immediately runs into contradiction since sum of such integrals is clearly zero, not $4 \pi g$ as it should be ( $g$ is the strength of the monopole). Thus the assumption fails and there must be a

${ }^{1}$ Work supported by the Department of Energy.

${ }^{2}$ In this talk I shall use the word "electron" to mean a charged spin- $\frac{1}{2}$ Dirac particle for simplicity. To describe a physical efectron-monopole system, one would need a quantum electrodynamics with monopoles, which at the present time has not been constructed satisfactorily. 


\begin{tabular}{|lll|}
\hline Difficulty: & $\begin{array}{l}\text { string singularity } \\
\text { Difficulty appears in: } \\
\text { electron's angular } \\
\text { wave function }\end{array}$ & $\begin{array}{c}\text { Lipkin-Weisberger-Peshkin difficulty } \\
\text { electron's radial wave function at the } \\
\text { origin }\end{array}$ \\
Resolution: & idea of section & $\begin{array}{c}\text { introduction of infinitesimal extra } \\
\text { magnetic moment of electron }\end{array}$ \\
\hline
\end{tabular}

Fig. 1. Difficulties associated with the electron-monopole system and their resolution.

singularity on that sphere. This happens for every sphere, so we have at least a string of singularities. If you have a charged particle in this field, this shows up in the angular part of its wave function.

The second type of difficulty occurs when you allow an electron to pass through the monopole. As is well known, this system possesses an extra piece - eg $\hat{r}$ for the angular momentum, which may be interpreted as the field angular momentum ( $\hat{r}$ here is the unit vector pointing to the electron from the monopole). Thus as the charge passes through the pole, this quantity suddenly changes sign, or to put it another way, this piece is undefined when they are on top of one another.

The above is a classical discussion. Quantum mechanically it was noted, in the study of the nonrelativistic Schrödinger equation, by Lipkin et al. (1969) as the failure of the Jacobi identity at the origin among the components of the momentum operator $\mathbf{p}-e \mathbf{A}$, viz.,

$$
\pi_{i} \equiv p_{i}-e A_{i} \quad\left[\pi_{1},\left[\pi_{2}, \pi_{3}\right]\right]+\text { cyclic }=-4 \pi e g \delta^{3}(\mathbf{r})
$$

This means that we will have trouble if the radial wave function of the electron does not vanish at the origin. Fortunately, for the nonrelativistic case this did not happen. But as we shall see later in the relativistic treatment of the system the situation actually prevails.

To sum up, there are two types of difficulties, namely, the string singularities and the Lipkin-Weisberger-Peshkin difficulty, which show up, respectively, in the angular and the radial wave functions of the charged particle.

These difficulties will be naturally resolved in the discussion to follow. The string singularities will be disposed of by the concept of the wave function as a section borrowed from the mathematics of fiber bundles, whereas the Lipkin-Weisberger-Peshkin difficulty will be resolved by the introduction of an infinitesimal extra magnetic moment of the electron (see Figure 1).

\section{BRIEF DESCRIPTION OF THE IDEA OF A SECTION}

First let me briefly discuss the resolution due to Wu and Yang (1975, 1976 ) of the first and the main difficulty. What Wu and Yang observed is the 


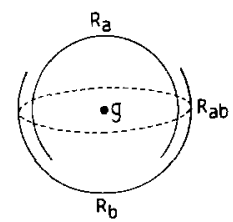

Fig. 2. Angular regions $R_{a}, R_{b}$, and $R_{a b}$ for the space around a monopole of strength $g$.

fact that the string singularity is just like a coordinate singularity which you encounter, for example, in trying to coordinatize a sphere by a single coordinate system. It arises from the global topology of a sphere. This situation, however, is quite familiar to mathematicians and the resolution is simply to cover the sphere or any given manifold by a sufficient number of coordinate patches with smooth coordinate transformations in the overlaps. All the global topological properties are now reflected in the nature of these transformations, i.e., how the coordinate patches are glued together.

This whole scheme fits into our situation very well. Consider dividing the space around the monopole into two overlapping regions $R_{a}$ and $R_{b}$ (see Figure 2). In $R_{a}$ we define

$$
\left(A_{u}\right)_{a}=g \frac{1-\cos \theta}{r \sin \theta} \hat{\varphi}
$$

This potential gives the correct magnetic field

$$
\mathbf{H}=g \frac{\hat{r}}{r^{3}}
$$

and is completely regular in $R_{a}$. Its only singularity is along the negative $z$ axis. In $R_{b}$ we define a different potential,

$$
\left(A_{\mu}\right)_{b}=-g \frac{(1+\cos \theta)}{r \sin \theta} \hat{\varphi}
$$

which also gives the same magnetic field (2.2). This is completely regular in $R_{b}$ with its only singularity along the positive $z$ axis. In the overlap, called $R_{a b}$, the difference is curl-less, hence a gradient, i.e., a gauge transformation. It is easily checked that

$$
\left(A_{\mu}\right)_{a}=\left(A_{\mu}\right)_{b}+2 g \partial_{\mu} \varphi=\left(A_{\mu}\right)_{b}+\frac{i}{e} S_{a b} \partial_{\mu} S_{a b}^{-1}
$$

where

$$
S_{a b}=e^{2 i e g \varphi}
$$

Now if you have a charged particle in this field, its wave function $\psi$ must also undergo a gauge transformation to preserve local gauge invariance, i.e.,

$$
\psi_{a}=S_{a b} \psi_{b}=e^{2 i e g \varphi} \psi_{b}
$$


Therefore the wave function must also be defined separately in regions $R_{a}$ and $R_{b}$. Notice that in (2.6) the single-valuedness of $\psi$ implies the single-valuedness of $S$, which in turn implies the famous Dirac quantization condition

$$
2 e g=\text { integer }
$$

Summarizing, (a) potential $A_{\mu} \equiv\left(\left(A_{\mu}\right)_{a},\left(A_{\mu}\right)_{b}\right)$ as a pair is completely free of singularities and (b) the wave function $\psi$ of the charged particle must also be defined in $R_{a}$ and $R_{b}$ separately so that in $R_{a b}, \psi_{a}=S_{a b} \psi_{b}$. Such a function is called a section in the mathematics of fiber bundles. We have thus successfully resolved the first difficulty with the aid of the concept of the wave function as a section.

\section{EIGENSECTIONS OF THE ANGULAR MOMENTUM OPERATOR-THE MONOPOLE HARMONICS}

In analyzing the wave equation of a charged particle in the presence of a monopole, one naturally needs eigenfunctions or rather eigensections of the angular momentum operator, which we shall call monopole harmonics $Y_{q, l, m}(\theta, \varphi)$ (Wu and Yang, 1976). They are generalizations of the ordinary spherical harmonics and carry an extra half-integer index $q=e g$ that specifies how the wave functions defined in the two regions $R_{a}$ and $R_{b}$ are glued together. A nonvanishing $q$, therefore, characterizes a nontrivial topology.

A. Spinless Case. If the charged particle is spinless, the angular momentum operator is of the form

$$
\mathbf{L}=\mathbf{r} \times(\mathbf{p}-e \mathbf{A})-q \hat{\mathbf{r}}
$$

Notice that $\mathbf{L}$ is defined in $R_{a}$ and $R_{b}$ separately due to the presence of $\mathrm{A}$. We define the monopole harmonics by

$$
\left.\begin{array}{l}
L^{2} Y_{q, l, m}=l(l+1) Y_{q, l, m} \\
L_{z} Y_{q, l, m}=m Y_{q, l, m}
\end{array}\right\} \text { in both } R_{a} \text { and } R_{b}
$$

The explicit form in $R_{a}$ is given by

$$
\begin{aligned}
\left(Y_{q, l, m}\right)_{a}= & 2^{m}\left[\frac{2 l+1}{4 \pi} \frac{(l-m) !}{(l+q) !} \frac{(l+m) !}{(l-q) !}\right]^{1 / 2} \\
& \times(1-x)^{\alpha / 2}(1+x)^{\beta / 2} P_{n}^{\alpha, \beta}(x) e^{i(m+q) \varphi}
\end{aligned}
$$

where $\alpha=-q-m, \beta=q-m, n=l+m, x=\cos \theta$, and $P_{n}^{\alpha, \beta}$ is the Jacobi polynomial given by

$$
P_{n}^{\alpha, \beta}(x)=\frac{(-1)^{n}}{2^{n} n !}(1-x)^{-\alpha}(1+x)^{-\beta} \frac{d^{n}}{d x^{n}}\left[(1-x)^{\alpha+n}(1+x)^{\beta+n}\right]
$$


$l$ here takes the values $|q|,|q|+1, \ldots$, etc., due to the presence of the field angular momentum. In $R_{b}$,

$$
\left(Y_{q, l, m}\right)_{b}=e^{-2 t q \varphi}\left(Y_{q, l, m}\right)_{a}
$$

The most important properties of $Y_{q, l, m}$ are:

(i) $Y_{q, l, m}$ as a section is everywhere analytic;

(ii) $Y_{q, l, m}$ for any fixed $q$ form a complete orthonormal system, i.e., one can expand any continuous section belonging to class $q$ in terms of $Y_{q, l, m}$.

B. Spin $-\frac{1}{2}$ Case. Generalization to the case for a particle with a spin $-\frac{1}{2}$ is straightforward. The total angular momentum is now given by

$$
\mathbf{J}=\mathbf{L}+\frac{1}{2} \boldsymbol{\sigma}
$$

For each value $j$ of the total angular momentum, one has two two-component natural eigensections $\varphi^{(1)}$ and $\varphi^{(2)}$, except for the lowest angular momentum case, for which there is only one. Explicitly, for $l=j-\frac{1}{2}$

$$
\varphi_{j m}^{(1)}=\left[\begin{array}{l}
\left(\frac{j+m}{2 j}\right)^{1 / 2} Y_{q, j-1 / 2, m-1 / 2} \\
\left(\frac{j-m}{2 j}\right)^{1 / 2} Y_{q, j-1 / 2, m+1 / 2}
\end{array}\right] \quad j=|q|+\frac{1}{2},|q|+\frac{3}{2}, \ldots
$$

for $l=j+\frac{1}{2}$

$$
\varphi_{3 m}^{(2)}=\left[\begin{array}{c}
-\left(\frac{j-m+1}{2 j+2}\right)^{1 / 2} Y_{q, j+1 / 2, m-1 / 2} \\
\left(\frac{j+m+1}{2 j+2}\right)^{1 / 2} Y_{q, j+1 / 2, m+1 / 2}
\end{array}\right] \quad j=|q|-\frac{1}{2},|q|+\frac{1}{2}, \ldots
$$

Since the state with the lowest possible angular momentum $\left(j=|q|-\frac{1}{2}\right)$ stands out, we give it a name

$$
\eta_{m} \equiv \varphi_{|q|}^{(2)}-1 / 2, m
$$

Again one can prove that $\left\{\varphi^{(1)}, \varphi^{(2)}\right\}$ and $\eta_{m}$ form an everywhere analytic complete orthonormal system of two-component eigensections.

\section{SOLUTIONS OF THE DIRAC EQUATION IN THE PRESENCE OF A MONOPOLE}

Having successfully resolved the first difficulty and with angular eigensections at hand, let us discuss the scattering of an electron off a fixed magnetic monopole. (In classical mechanics it was first discussed by Poincaré (1896). In the context of nonrelativistic quantum mechanics, it was first discussed by 
Banderet (1946). See also Harish-Chandra (1948), A. S. Goldhaber (1965), Schwinger et al. (1976), and Boulware et al. (1976).) In the course of the discussion we shall encounter the second difficulty.

The wave equation for stationary states is of a familiar form:

$$
\left[\begin{array}{ll}
-E+m & \boldsymbol{\sigma} \cdot(\mathbf{p}-e \mathbf{A}) \\
\boldsymbol{\sigma} \cdot(\mathbf{p}-e \mathbf{A}) & -E-m
\end{array} \psi \mathbf{0}=0\right]
$$

We shall, as usual, decompose $\psi$ into partial waves and then solve the radial equations. This may be done very simply by noting that the operators $\sigma \cdot \hat{r}$ and $\sigma \cdot(\mathbf{p}-e \mathbf{A})$ are still rotation invariant even in the presence of a monopole. This means that these operators cannot change the values of $j$ and $m$ and hence can at most mix two basis eigensections $\varphi^{(1)}$ and $\varphi^{(2)}$ for given $j$ and $m$, i.e., they have $2 \times 2$ matrix representations in this basis. It turns out that it is extremely convenient to define a new basis $\xi^{(1)}$ and $\xi^{(2)}$ by a spinor rotation, viz.,

$$
\left(\xi_{j m}^{(1)}, \xi_{j m}^{(2)}\right)=\left(\varphi_{j m}^{(1)}, \varphi_{j m}^{(2)}\right) e^{i(\alpha / 2) \sigma_{y}}
$$

with $\sin \alpha=q /\left(j+\frac{1}{2}\right)$.

In this basis

$$
(\boldsymbol{\sigma} \cdot \hat{r})\left(\xi_{j m}^{(1)}, \xi_{j m}^{(2)}\right)=\left(\xi_{j m}^{(1)}, \xi_{j m}^{(2)}\right)\left(-\sigma_{x}\right)
$$

i.e., $\sigma \cdot \hat{r}$ is precisely $-\sigma_{x}$, just like the familiar hydrogen atom case. Rewriting this as

$$
\boldsymbol{\sigma} \cdot \hat{r}\left(\xi^{(1)} \mp \xi^{(2)}\right)= \pm\left(\xi^{(1)} \mp \xi^{(2)}\right)
$$

one finds that the simple combinations $\xi^{(1)} \mp \xi^{(2)}$ are the eigenstates of $\sigma \cdot \hat{r}$, which is the helicity operator for large $r$ for the outgoing wave, with eigenvalues \pm 1 . For the state $\eta_{m}$ with the lowest angular momentum, it is even simpler since there is no other state to mix into and one finds

$$
\sigma \cdot \hat{r} \eta_{m}=\frac{q}{|q|} \eta_{m}
$$

Analysis is entirely similar for the operator $\sigma \cdot(\mathbf{p}-e \mathbf{A})$ with the result

$$
\begin{aligned}
& \boldsymbol{\sigma} \cdot(\mathbf{p}-e \mathbf{A}) f(r) \xi_{j m}^{(1)}=i\left(\partial_{r}+\frac{1-\mu}{r}\right) f(r) \xi_{j m}^{(2)} \\
& \boldsymbol{\sigma} \cdot(\mathbf{p}-e \mathbf{A}) g(r) \xi_{j m}^{(2)}=i\left(\partial_{r}+\frac{1+\mu}{r}\right) f(r) \xi_{j m}^{(1)} \\
& \boldsymbol{\sigma} \cdot(\mathbf{p}-e \mathbf{A}) h(r) \eta_{m}=\frac{1}{i} \frac{q}{|q|}\left(\partial_{r}+\frac{1}{r}\right) h(r) \eta_{m}
\end{aligned}
$$

where $\mu=\left[\left(j+\frac{1}{2}\right)^{2}-q^{2}\right]^{1 / 2}$. 
With (4.4), (4.5), and (4.6) one can immediately write down the radial equations and solve them exactly. There are three types of solutions, which may be summarized as follows:

(i) $j \geqslant|q|+\frac{1}{2}$

$$
\begin{aligned}
\text { Type (1) } \quad \psi_{j m}^{(1)} & =\left[\begin{array}{l}
f(r) \xi_{m}^{(1)} \\
g(r) \xi_{j m}^{(2)}
\end{array}\right] \\
f(r) & =j_{u-1}(k r) \quad g(r)=\frac{1}{i} \frac{k}{E+m} j_{\mu}(k r)
\end{aligned}
$$

Type (2) $\quad \psi_{j m}^{(2)}=\left[\begin{array}{l}f(r) \xi_{j m}^{(2)} \\ g(r) \xi_{j m}^{(1)}\end{array}\right]$

$$
f(r)=j_{\mu}(k r) \quad g(r)=i \frac{k}{E+m} j_{\mu-1}(k r)
$$

(ii) $j=|q|+\frac{1}{2}$

$$
\begin{aligned}
\text { Type (3) } \quad \psi_{j m}^{(3)} & =\left[\begin{array}{l}
f(r) \eta_{m} \\
g(r) \eta_{m}
\end{array}\right] \\
f(r) & =\frac{\sin (k r+\delta)}{k r} \quad g(r)=\frac{1}{i} \frac{k}{E+m} \frac{\cos (k r+\delta)}{k r}
\end{aligned}
$$

Here $j_{\mu}(k r)$ is the spherical Bessel function of order $\mu$ [defined in equation (4.6)] and $\delta$ is a phase to be discussed.

It is at this stage that one encounters the Lipkin-Weisberger-Peshkin difficulty. While type (1) and (2) radial functions vanish at the origin, type (3) radial functions do not, and in fact at least one of them blows up. This means that the electron in this state goes through the monopole. Thus we find that our naive Hamiltonian is ill defined. To avoid the difficulty, we must modify our Hamiltonian so that the radial functions all vanish at the origin. Fortunately there is a natural solution to this problem. ${ }^{3}$ By endowing an infinitesimal extra magnetic moment $\kappa e / 2 m$ to the electron, one can make wave functions vanish at the origin regardless of the sign of $\kappa$. Our new Hamiltonian is now

$$
H_{\text {new }}=H_{\text {old }}-\frac{\kappa q}{2 m} \beta \frac{\boldsymbol{\Sigma} \cdot \hat{r}}{r^{2}}
$$

where $\beta$ and $\boldsymbol{\Sigma}$ are the usual Dirac matrices. Notice that (a) the added term falls off like $1 / r^{2}$ and the large $r$ behavior of the radial functions are unmodified, and (b) for $j \geqslant|q|+\frac{1}{2}$, although the $\beta$ matrix mixes the type (1) and (2)

${ }^{3}$ It was shown by A. S. Goldhaber that the following is the unique way of resolving the problem if one insists on the natural transformation properties of the system under $C, P$, and $T$ operations. [See Goldhaber (1977) for detail.] 


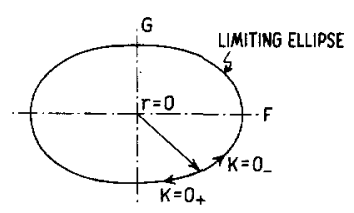

Fig. 3. The limiting ellipse and the behavior of $F$ and $G$ are explained in the text.

solutions, the radial wave functions will remain at zero at the origin, and as $\kappa \rightarrow 0$ the mixing is removed. That is, there is no influence upon type (1) and (2) solutions as $\kappa \rightarrow 0$.

Now let us look closely at the troublesome case of the state with the lowest angular momentum. Defining the $F$ and $G$ functions by

$$
f=\frac{\kappa q}{|\kappa q|} \frac{1}{r} F \quad g=\frac{1}{i} \frac{1}{r} G
$$

the radial equations take the form

$$
\frac{d F}{d r}=\left[(E+m) \frac{\kappa}{|\kappa|}-\frac{|\kappa q|}{2 m} \frac{1}{r^{2}}\right] \quad \frac{d G}{d r}=\left[-(E-m) \frac{\kappa}{|\kappa|}-\frac{|\kappa q|}{2 m} \frac{1}{r^{2}}\right] F
$$

By looking at the small $r$ behavior, which is of our interest, we easily find that $F$ and $G$ strongly go to zero as $r \rightarrow 0$ with their ratio approaching -1 . Namely,

$$
\left.\begin{array}{rl}
-G \sim F \propto \exp \left(-\frac{|\kappa q|}{2 m} \frac{1}{r}\right) & \rightarrow 0 \\
G / F & \rightarrow 1
\end{array}\right\} \text { as } r \rightarrow 0
$$

In more geometrical terms the situation is as follows (see Figure 3). In the $F$ - $G$ plane, the solution starts from the origin at $r=0$ and for large $r$ describes an ellipse since the large- $r$ behavior has not changed. As $\kappa$ becomes smaller and smaller, the limiting ellipse is approached earlier and earlier, and finally in the limit $k \rightarrow 0$, the situation looks like that of Figure 3.

In any case, the second difficulty is now removed and the correct boundary condition that fixes the phase $\delta$ completely is given by (4.13). The determined phase is of the form

$$
\delta=\delta_{3} \equiv \delta \frac{\kappa}{|\kappa|} \frac{\kappa}{E+m}
$$

Notice that it depends on the energy and the sign of $\kappa$.

\section{WAVE FUNCTIONS DESCRIBING SCATTERING}

Having resolved the second difficulty successfully, we may now easily construct the scattering solution. 
Let us send an incident plane wave of positive or negative helicity

$$
\begin{aligned}
& \left(\frac{E+m}{2 E}\right)^{1 / 2} e^{-i k z}\left[\begin{array}{c}
0 \\
1 \\
0 \\
k \\
\hline E+m
\end{array}\right] \quad(h=+1) \\
& \left(\frac{E+m}{2 E}\right)^{1 / 2} e^{-i k z}\left[\begin{array}{c}
1 \\
0 \\
-k \\
E+m \\
0
\end{array}\right] \quad(h=-1)
\end{aligned}
$$

along the negative $z$ direction. These are essentially two-component wave functions. Recalling that $\left\{\xi^{(1)}, \xi^{(2)}\right\}$ and $\eta_{m}$ form a complete orthonormal system, we can expand the incident wave in terms of them. The incoming part of the incident wave may be expanded as

$$
\begin{aligned}
\left(\begin{array}{l}
0 \\
1
\end{array}\right) & \\
e_{\mathrm{inc}}^{-i k z} \text { or }= & -\frac{e^{-i k r}}{2 i k r} 2 \pi^{1 / 2} \\
\left(\begin{array}{l}
1 \\
0
\end{array}\right) & \\
& \times\left.\left\{ \pm(|q| \mp q)^{1 / 2} \eta_{m}+\sum_{j=|q|+1 / 2}^{\infty}\left(j+\frac{1}{2}\right)^{1 / 2}\left(\xi_{m j}^{(1)} \pm \xi_{j m}^{(2)}\right)\right\}\right|_{m=-q \neq(1 / 2)}
\end{aligned}
$$

where the upper (lower) sign refers to $h=+1(-1)$. Notice that $\xi^{(1)} \pm \xi^{(2)}$ and $\eta_{m}$ appear as they should, since we are expanding a helicity eigenstate. Matching this to the incoming part of the most general solution, i.e., the superposition of type (1), (2), and (3) solutions, one obtains the exact scattering wave function, whose outgoing part is given by

$$
\begin{aligned}
{ }^{ \pm} \psi_{\text {out }}= & \frac{e^{i k r}}{2 i k r}\left(\frac{E+m}{2 E}\right)^{1 / 2} 4 \pi^{1 / 2} \\
& \times\left\{ \pm(|q| \mp q)^{1 / 2} e^{2 i \delta_{3}} \eta_{m}\left[\begin{array}{c}
1 \\
\frac{q}{|q|} \frac{k}{E+m}
\end{array}\right]\right. \\
& \left.-\sum_{j=|q|+1 / 2}^{\infty} e^{-i \pi \mu}\left(j+\frac{1}{2}\right)^{1 / 2}\left(\xi_{j m}^{(1)} \mp \xi_{j m}^{(2)}\right)\left[\begin{array}{c}
1 \\
\pm \frac{k}{E+m}
\end{array}\right]\right\}\left.\right|_{m=-q \neq(1 / 2)}
\end{aligned}
$$


where the superscripts \pm on $\psi_{\text {out }}$ refer to the initial helicities. Note that again only $\xi^{(1)} \mp \xi^{(2)}$ and $\eta_{m}$ appear and the above expression is already essentially helicity decomposed. Defining the helicity flip (HF) and the helicity nonflip (HNF) amplitudes by

$$
{ }^{+} \psi=C^{+\rightarrow+} \chi^{+}+C^{+\rightarrow-} \chi^{-} \quad-\psi=C^{-\rightarrow+} \chi^{+}+C^{-\rightarrow-} \chi^{-}
$$

where $\chi^{ \pm}$are the normalized helicity eigenstates, one can read off the HF and HNF amplitudes immediately:

HF: $\begin{aligned} C^{+\rightarrow-} & = \begin{cases}0 & \text { for } q>0 \\ \frac{e^{i k r}}{2 i k r} 2 q\left(\sin \frac{\Theta}{2}\right)^{2|q|-1} e^{-i \varphi+2 i \delta_{3}} & \text { for } q<0\end{cases} \\ C^{-\rightarrow+} & = \begin{cases}\frac{e^{i k r}}{2 i k r} 2 q\left(\sin \frac{\Theta}{2}\right)^{2|q|-1} e^{+i \varphi+2 i \delta_{3}} & \text { for } q>0 \\ 0 & \text { for } q<0\end{cases} \end{aligned}$

HNF: $\quad C^{+\rightarrow+}=C^{-\rightarrow-}=-\frac{e^{i k r}}{2 i k r} T_{q}(\Theta)$

$$
\begin{aligned}
T_{q}(\Theta)= & (2 \pi)^{1 / 2} \frac{1}{\sin (\Theta / 2)} \sum_{j=|q|+1 / 2}^{\infty} \mu e^{-i \pi \mu} \\
\times & {\left[\frac{1}{j^{1 / 2}} Y_{q, j-(1 / 2),-q}(\pi-\Theta, \varphi)\right.} \\
& \left.-\frac{1}{(j+1)^{1 / 2}} Y_{q, j+(1 / 2),-q}(\pi-\Theta, \varphi)\right]
\end{aligned}
$$

where $\Theta$ is the scattering angle.

The salient features of this result may be stated as follows.

(a) The helicity flip amplitude consists entirely of the partial wave with the lowest angular momentum as you may see from the appearance of the phase $\delta_{3}$. The amplitude depends on the initial helicity $h_{\mathrm{n}}$ and vanishes if $h_{\mathrm{in}} q>0$. Notice that for large $q$ the amplitude is highly backward peaked. These are the simple consequences of the angular-momentum conservation. Further, $\left|C^{+-}(q)\right|$ is equal to $\left|C^{-+}(-q)\right|$. This may be understood from the invariance property of the system under $C, P, T$ operations. $^{4}$

(b) The helicity nonflip amplitude, on the other hand, consists entirely of higher partial waves. It involves a complicated infinite series of monopole harmonics with a cumbersome phase factor. For large $\Theta$, the series must be summed numerically. For small $\Theta, T_{q}(\Theta)$ is forward divergent, just like the

${ }^{4}$ For more detailed discussion, see Goldhaber (1977). 
Rutherford cross section, and one can isolate and sum the forward divergent terms exactly. This gives

$$
\begin{aligned}
T_{q}(\Theta)= & -q e^{-i \pi q} \frac{1}{\sin ^{2}(\Theta / 2)} \\
& \times\left\{\frac{\cos (\Theta / 2)}{1+\sin (\Theta / 2)}\left[1+\left(1+\frac{i \pi q}{2}\right) \sin \frac{\Theta}{2}\right]\right. \\
& \left.+\frac{1}{2}\left(1+i \pi q-\frac{\pi^{2} q^{2}}{4}\right) \frac{1-[\sin (\Theta / 2)]^{2 q}}{\cos (\Theta / 2)} \sin ^{2} \frac{\Theta}{2}\right\}
\end{aligned}
$$

+ forward finite terms

$$
\sim-q e^{-i \pi q} \frac{1}{\sin ^{2}(\Theta / 2)} \text { for small } \Theta
$$

To illustrate, I will show the behavior obtained by numerical analysis (Figure 4). Plotted here are the ratios of the electron-monopole scattering cross section (unpolarized electron beam) for two values of $q$ to the Rutherford cross section, except for the velocity-dependent constant factor (solid lines). For comparison we have also plotted the same ratios for the case of spinless nonrelativistic charged particle (dotted lines) obtained by Banderet (1946). In the forward direction, there is not much difference from the Rutherford cross section. The enhancement in the backward direction is due to the HF amplitude given by the lowest partial wave.

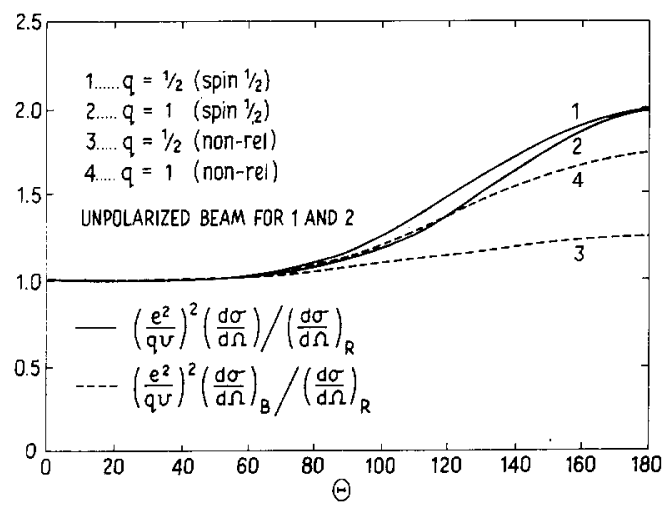

Fig. 4. Ratio of monopole to Rutherford cross sections. $d \sigma / d \Omega$ is the cross section for scattering of a Dirac particle of charge $e$ by a fixed monopole $g .(d \sigma / d \Omega)_{B}$ is the cross section for scattering of a nonrelativistic spinless particle of charge $e$ by a fixed monopole $g .(d \sigma / d \Omega)_{R}$ is the Rutherford cross section for' scattering of a particle of charge $e$ by a fixed target of charge $e: q=e g, \Theta$ is the scattering angle in degrees. 


\section{EXISTENCE OF BOUND STATES}

Having understood the scattering properties, let us go on to the question of bound states.

First it is instructive to ask whether one can have bound states without the extra magnetic moment, i.e., in the limit $\kappa \rightarrow 0$. Since in this case we have the exact solutions [equations (4.7), (4.8), and (4.9)], we just replace the momentum $k$ by $i k$ and see if we get normalizable solutions. For $j \geqslant|q|+\frac{1}{2}$, the solutions, if you recall, are spherical Bessel functions of argument $k r$. Inserting an $i$ gives modified Bessel functions, which, if you choose the ones with exponential tails, are not normalizable around the origin. For $j=$ $|q|-\frac{1}{2}$, the trigonometric functions become real exponential functions and one can make a bound state. So for $\kappa \rightarrow 0$, there exist no bound states except for the $j=|q|-\frac{1}{2}$ state. But we have learned that the extra magnetic moment strongly damps the wave functions at the origin. Does this cure the bad behavior at the origin and give us more bound states? The answer turns out to be yes and we find at least two classes of infinitely many bound states.

Let us quickly see what types of equations we must analyze. The equation for the stationary state is of the form

$$
\left[\begin{array}{ll}
-E+M-\frac{\kappa g}{2 M} \frac{\boldsymbol{\sigma} \cdot \hat{r}}{r^{2}} & \boldsymbol{\sigma} \cdot(\mathbf{p}-e \mathbf{A}) \\
\boldsymbol{\sigma} \cdot(\mathbf{p}-e \mathbf{A}) & -E-M+\frac{\kappa g}{2 M} \frac{\boldsymbol{\sigma} \cdot \hat{r}}{r^{2}}
\end{array}\right] \psi=0
$$

Now there are two types of solutions:

(i) Type $\mathrm{A}, j \geqslant|q|+\frac{1}{2}$ :

$$
\psi=\frac{1}{r}\left[\begin{array}{c}
h_{1}(r) \xi_{j m}^{(1)}+h_{2}(r) \xi_{j m}^{(2)} \\
\frac{1}{i} \frac{\kappa q}{|\kappa q|}\left[h_{3}(r) \xi_{j m}^{(1)}+h_{4}(r) \xi_{j m}^{(2)}\right]
\end{array}\right]
$$

(ii) Type $\mathrm{B}, j=|q|-\frac{1}{2}$ :

$$
\psi=\frac{1}{r}\left[\begin{array}{c}
\frac{\kappa q}{|\kappa q|} F(r) \eta_{m} \\
\frac{1}{i} G(r) \eta_{m}
\end{array}\right]
$$

For ease of analysis, introduce dimensionless variables

$$
\rho \equiv \frac{2 M}{|\kappa q|} r \quad A_{0} \equiv \frac{\kappa q}{2} \quad A \equiv \frac{\kappa|q|}{2} \quad B_{0} \equiv \frac{\kappa q}{2} \frac{E}{M} \quad B \equiv \frac{\kappa|q|}{2} \frac{E}{M}
$$


Then the radial equations take the form

Type A: $\left[\begin{array}{cccc}\partial_{\rho}-\frac{\mu}{\rho} & 0 & \frac{1}{\rho^{2}} & A_{0}+B_{0} \\ 0 & \partial_{\rho}+\frac{\mu}{\rho} & A_{0}+B_{0} & \frac{1}{\rho^{2}} \\ \frac{1}{\rho^{2}} & A_{0}-B_{0} & \partial_{\rho}-\frac{\mu}{\rho} & 0 \\ A_{0}-B_{0} & \frac{1}{\rho^{2}} & 0 & \partial_{\rho}+\frac{\mu}{\rho}\end{array}\right]\left[\begin{array}{l}h_{1} \\ h_{2} \\ h_{3} \\ h_{4}\end{array}\right]=0$

Type B: $\quad \frac{d F}{d \rho}=\left(A+B-\frac{1}{\rho^{2}}\right) G \quad \frac{d G}{d \rho}=\left(A-B-\frac{1}{\rho^{2}}\right) F$

with the boundary conditions that $h_{i}(i=1,2,3,4), G$, and $F$ all vanish at $\rho=0$ and $\infty$. Neither of these equations is, in general, exactly soluble.

The first set of infinite number of bound states consists of those with $E=0$ for each value of angular momentum for any value of $\kappa$. If we put $E=0$ (i.e., $B=0$ ) in (6.5) and (6.6) the equations decouple nicely and one can solve them exactly with the following results:

Type A: $\quad h_{1}=-h_{3}=\left(\frac{r}{2}\right)^{1 / 2} \exp \left(-\frac{|\kappa q|}{2 M r}\right) K_{\mu-1 / 2}(M r)$
$h_{2}=-h_{4}=-\frac{q}{|q|}\left(\frac{r}{2}\right)^{1 / 2} \exp \left(-\frac{|\kappa q|}{2 M r}\right) K_{u+1 / 2}(M r)$

Type B: $\quad F=-G=\frac{1}{2^{1 / 2}} \exp \left(-\frac{|\kappa q|}{2 M r}-M r\right) \underset{\kappa \rightarrow 0}{\longrightarrow} \frac{1}{2^{1 / 2}} \exp (-M r)$

$K_{u}$ is the modified $K$-Bessel function of order $\mu$. Notice that, as we expected, the nonvanishing $\kappa$ strongly suppresses the divergent behavior of the $K$-Bessel function at the origin and that for the type B case the $\kappa \rightarrow 0$ limit still gives a bound state.

The analysis for the $E \neq 0$ case is more complicated. Especially for type A states, equation (6.5) has so far been intractable. For type B states, although we may not solve the equation in a closed form, we can nevertheless analyze it satisfactorily. Let me quickly describe the outline of it and convince you that under certain conditions there exist another set of infinite number of bound states for this particular value of the angular momentum.

First let us make two simple observations. (a) The bound state spectrum, if it exists, is symmetric about $E=0$. (b) If $|A|<|B|$, i.e., if $M<|E|$, the large- $\rho$ behavior is oscillatory and there are no bound states. Thus we shall restrict ourselves to the case where $|A|>B>0$ holds. 


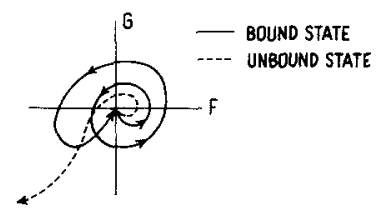

Fig. 5. Typical bound and unbound states in $G-F$ plane.

The method of analysis is to study the behavior of the integral in the $F-G$ plane just like for the familiar Sturm-Liouville problem. An integral corresponding to a bound state starts off at the origin at $r=0$ and comes back to the origin as $r \rightarrow \infty$ (see Figure 5). The number of times it winds around will be the number of nodes and so forth. In contrast, an integral corresponding to an unbound state does not come back to the origin as $r \rightarrow \infty$. Thus we shall concentrate on how the integral winds about the origin. For this purpose it is convenient to use a polar representation

$$
F=R \cos \left(\frac{\varphi}{2}-\frac{\pi}{4}\right) \quad G=R \sin \left(\frac{\varphi}{2}-\frac{\pi}{4}\right)
$$

This choice of the angle is convenient since the boundary condition at the origin turns out to be simply $\varphi(\rho=0)=0$. Eliminating $R$ by taking the ratio of $F$ and $G$, one obtains a nonlinear first-order differential equation for the angle $\varphi$, viz.,

$$
\frac{d \varphi}{d \rho}=-2 B+2\left(A-\frac{1}{\rho^{2}}\right) \sin \varphi \equiv T(A, B, \rho)
$$

We shall hereafter concentrate on the case where $A>B>0$ (i.e., $\kappa>0$ ), since for $\kappa<0$ it can be proved, by the similar arguments we will go through, that there are no bound states.

We first study the sign of the derivative $d \varphi / d \rho$ to see how $\varphi$ increases or decreases. So we plot the sign of $T(A, B, \rho)$ in the $\varphi-\rho$ plane (Figure 6). There are, of course, two types of regions: the - region where $\varphi$ is decreasing and

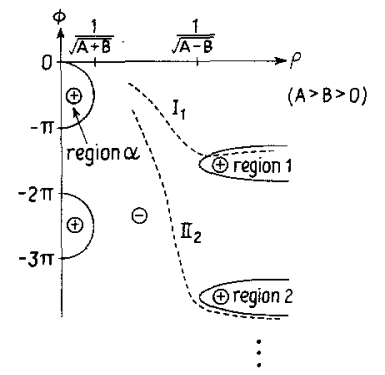

Fig. 6. Sign of derivative $d \varphi / d \rho$ [i.e., $T(A, B, \rho)$ in equation (6.10)] in the $\varphi-\rho$ plane for $A>B>0$. 
the + region where $\varphi$ is increasing. Along the boundaries of these two regions, $\varphi^{\prime}$ vanishes so the integral must cross these boundaries horizontally. One remark is in order before we proceed. The solution with zero energy that we found before corresponds to the $\varphi \equiv 0$ line in this picture. Since the integrals cannot cross each other, they must lie completely above or below this line. By looking at the small- $\rho$ behavior, one finds that $\varphi$ is always negative. Further, the region $\alpha$ turns out to be irrelevant to the analysis.

Now an interesting thing to observe is that the regions $1,2, \ldots$ are "trapping" regions in the following sense. Because of their shapes, once an integral crosses this border, it can never get out. It keeps increasing in this region and can never cross the border again horizontally. So $\varphi$ will either get trapped and keep increasing or not get trapped and keep decreasing. In either case, $\varphi(\rho)$ is monotonic for large $\rho$ and hence the $\lim _{\rho \rightarrow \infty} \varphi(\rho)$ exists (including $-\infty)$. The key idea now is that we can classify the integrals by the values of $\varphi(\infty)$.

There are essentially two classes (see Figure 7 ). To class $I_{n}$ belong all the integrals which get trapped in the region $n$. For this class one can show that $F$ and $G$ exponentially diverge as $\rho \rightarrow \infty$. So this class is not a class for bound states. Another type of class is called class $\mathrm{II}_{n}$, to which belong integrals that barely touch the lower lip of the $n$th trapping region and approach to a certain characteristic value of $\varphi(\infty)$ from above. For this, one can prove that $F$ and $G$ are both exponentially decreasing at large $\rho$. This is the class for the bound states.

Now the question is, is class $\mathrm{II}_{n}$ realized at some energy, i.e., at some $B$ ? Thus we must study the $B$ dependence of $\varphi(\infty)$. The key theorem, which is easy to prove, is

$$
\frac{\partial \varphi(\rho)}{\partial B}<0
$$

at any fixed $\rho$. Taking the limit of this statement, we get

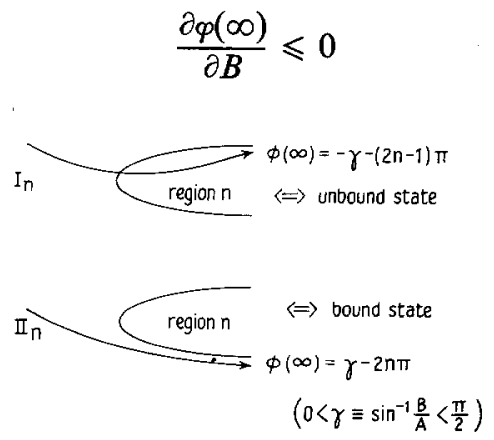

Fig. 7. Two classes of the behavior of $\varphi(\rho)$. 


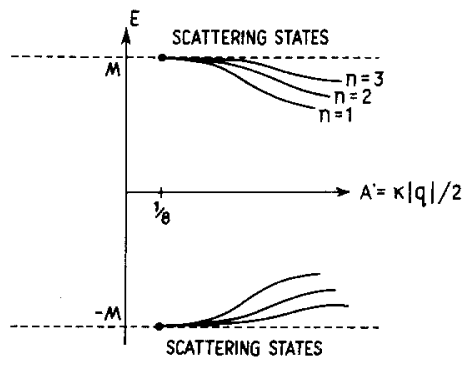

Fig. 8. Bound-state energy level spectrum as a function of $A=k|q| / 2$. Levels labeled by $n=4.5, \ldots$ are not shown.

i.e., $\varphi(\infty)$ is a nonincreasing function of $B$. Thus as $B$ increases, two things happen: $\varphi$ goes down at every finite $\rho$, and the trapping regions recede to the right to give more room, so to speak, for $\varphi$ to decrease. Therefore as $B$ increases one may expect the integral to descend from region 1 to region 2 , to region 3, etc. If this is the case, class $\mathrm{II}_{n}$ for every $n$ will be realized at some values of $B$. To see whether this really happens we study the limit $B \rightarrow A$. Equation (6.6) for the limiting case can be solved exactly and we find the following results: If $\kappa|q| \leqslant \frac{1}{4}$, the integral is trapped in region 1 for all values of $B$ and there exist no bound states.

Let me now summarize our findings.

(i) For each $j=|q|-\frac{1}{2},|q|+\frac{1}{2}, \ldots$, there exists a bound state with $E=0$ for any $\kappa \neq 0$. For $j=|q|-\frac{1}{2}$, the $\kappa \rightarrow 0$ limit still yields a bound state.

(ii) For $j=|q|-\frac{1}{2}$, there exist countably infinite number of bound states with $E \neq 0$ if $\kappa|q|>\frac{1}{4}$. The energy spectrum is symmetric about $E=0$. If $\kappa|q| \leqslant \frac{1}{4}$, there is no bound state with $E \neq 0$.

The spectrum is sketched in Figure 8. For very loosely bound states, i.e., for large $n$, one can compute the leading term for the expression of the bound-state energy. It is of the form

$$
1-\frac{E_{n}}{m} \sim \exp \frac{-4 \pi n}{(4 \kappa|q|-1)^{1 / 2}} \text { as } n \rightarrow \infty \text { with } \kappa|q| \text { fixed }
$$

Finally, let me make the following remarks. ${ }^{5}$

(a) Firstly, the existence of the bound states with $E=0$ implies electronpositron plasma structure around the monopole. Suppose you create an $e^{+} e^{-}$ pair from the vacuum, costing energy of $2 M$. Binding them to the monopole

${ }^{5}$ Lacking a consistent theory of quantum electrodynamics of electrons and monopoles, it is difficult to predict how these remarks will be subject to quantum field theoretical corrections. One may, however, expect the qualitative properties of tightly bound states to survive such corrections. 
in the above states you gain energy of $2 M$ plus a little extra due to the Coulombic interaction between them. Therefore it is energetically more favorable to create $e^{+} e^{-}$pairs out of the vacuum and bind them to the monopole. One can do this for each value of angular momentum so one would have a plasma of electrons and positrons around the monopole.

(b) Secondly, I remark on the permanent electric dipole moment of the bound system. It is well known that this system violates both $T$ and $P$ invariance because the monopole field has "wrong" transformation properties under $T$ and $P$. As a result it is possible that the bound states of the system may possess a permanent electric dipole moment usually forbidden due to $T$ or $P$ invariance. This conjecture is indeed correct (Kazama, 1977) and, for example, a loosely bound state with the lowest angular momentum possesses a large electric dipole moment which depends exponentially on the principal quantum number $n$, viz.,

$$
\left|d_{z}\right|=\frac{m}{|q|+\frac{1}{2}} \frac{e}{2 M} \exp \frac{m}{(4|\kappa q|-1)^{1 / 2}}
$$

\section{REFERENCES}

Banderet, P. P. (1946). Helvetica Physica Acta, 19, 503.

Boulware et al. (1976). Physical Review D, 14, 2708.

Dirac, P. A. M. (1931). Proceedings of the Royal Society of London, Series A, 133, 60; (1948). Physical Review, 74, 817.

Goldhaber, A. S. (1965). Physical Review, 140, B1407; (1977). ITP-5B-77-29.

Harish-Chandra (1948). Physical Review, 74, 883.

Kazama, Y. (1977). Physical Review D, 15, 3078.

Kazama, Y., Yang, C. N., and Goldhaber, A. S. (1977). Physical Review D, 15, 2287.

Kazama, Y., and Yang, C. N. (1977). Physical Review D, 15, 2300.

Lipkin, H. J., Weisberger, W. I., and Peshkin, M. (1969). Annals of Physics (New York), $53,203$.

Poincaré, H. (1896). Comptes Rendus, 123, 530.

Schwinger, J., Milton, K. A., Tsai, W. Y., De Raad, Jr., L. L., and Clark, D. C. (1976). Annals of Physics (New York), 101, 451.

Wu, T. T., and Yang, C. N. (1975). Physical Review D, 12, 3845; (1976). Nuclear Physics, B107, 365. 